\section{Habronema e Draschia: alguns dados sobre infecções em eqüinos, asininos e muares de alguns estados brasileiros*}

\author{
Habronema and Draschia: occurrences in equines, \\ asinines and mules from some brazilian states
}

\author{
Amália Verônica Mendes SILVA'; Claudia Freire de Andrade MORAES'; \\ Helio Martins de Araújo COSTA
}

CORRESPONDENCE TO: Hélio Martins de Araújo Costa Departamento de Parasitologia Instituto de Cièncias Biológicas UFMG Av. Antonio Carlos, 6627 - Pampulha 31270-901 - Belo Horizonte - MG Brasil
1. Universidade Federal de Minas Gerais - MG

2. Escola de Veterinária da Universidade Federal de Minas Gerais - $M G$

3- Instituto de Ciências Biológicas Universidade Federal de Minas Gerais - MG

\title{
RESUMO
}

Foram examinados os estômagos de 37 Equus caballus, 18 Equus asinus e 22 muares, procedentes do Estado do Maranhão, Piauí, Ceará, Rio Grande do Norte, Paraiba, Pernambuco, Minas Gerais e Goiás, para a pesquisa de espirurídeos e foram encontrados: Draschia megastoma (Rudolphi, 1819), Habronema muscae (Carter, 1861) e Habronema microstoma (Schneider, 1866). Foram consideradas as prevalências, as intensidades médias e as relações Macho/Fêmea para estes parasitos, nos três hospedeiros. A análise de variância mostrou não ocorrerem diferenças estatisticamente significativas entre as intensidades médias de $H$. muscae e $H$. microstoma, inclusive na comparação entre os hospedeiros.

UNITERMOS: Habronema; Draschia; Equidae; Mulas: Brasil.

\section{INTRODUCĀO}

$\mathrm{N}$ os gêneros Hahronema e Draschia encontramos espécies parasitas de equídeos com ampla distribuição geográfica. Os adultos de Habronema ocasionam gastrite catarral, enquanto Draschia megastoma pode dar origem a formações tumorais na parede do estômago, no interior das quais são encontrados os exemplares do parasito. São espécies de grande interesse para a Medicina Veterinária, sobretudo pela possibilidade de as moscas, seus hospedeiros intermediários, deixarem as larvas em feridas abertas, assim dando origem à habronemose cutânea, que algumas vezes tem levado à inutilização de animais para o trabalho. Apesar desta importância, os dados existentes em nossa literatura dizem respeito, principalmente, a registros de achados fortuitos. Enquanto isso, em outros países, alguns pesquisadores têm demonstrado maior preocupação, investigando as ocorrências e as intensidades médias das infecções.

Foster; Ortiz' (1937), em estudo sobre parasitos de eqüinos no Panamá, assinalaram a presença de $H$. megastoma em $8 \%$, com a média de quatro exemplares, $H$. muscac em $74 \%$, com a média de 21 espécimes e $H$. microstoma em $62 \%$, com a média de 15 exemplares.

Christensen; Roth' (1942), estudando a gastrite parasitária em cavalos na Dinamarca, assinalaram a presença de

* Projeto FINEP
Habronema spp. (H. muscae e/ou H. microstoma) em 45\% dos casos.

Waddell ${ }^{2 n}$ (1969), em Brisbane (Austrália), após o exame de 280 estômagos de cavalos, assinalou a presença de $\boldsymbol{H}$. megastoma em $41 \%$ e H. muscae em $72 \%$, (iraber" (1970), em necrópsias de 183 asininos e 51 eqüinos na República do Tchad (Árica), assinalou a presença de $H$. megastoma em $53,3 \%$ e $9,8 \%$, respectivamente, $H$. microstoma em $14,7 \%$ e $7,84 \%$; e H. muscae em 20,2 e $27,45 \%$, com as intensidades médias de $171(10-2.500)$ e $186(30-500)$ de $H$. megastoma, de $28(1-120)$ de 1 . microstoma em asininos e de $98(1-902)$ e $345(15-1.000)$ de $H$. muscae, respectivamente, para asininos e eqüinos.

Scialdo $^{18}$ (1977), após o exame de 200) estômagos de cavalos procedentes de estados das regiões Sudoesie, Sudeste e Central dos Estados Unidos, assinalou $/ 1$. muscae em $11 \%$ e H. microstoma em $24 \%$.

Ouhelli et al. ${ }^{13}(1979)$ examinaram os estômagos de 86 eqüinos no Marrocos e assinalaram a presença de $I$. microstoma e H. muscae.

Alcaino et al.' (1980) estudaram os estômagos de 419 eqüinos no Chile e encontraram $22,9 \%$ parasiliados por $H$. megastoma e $17,7 \%$ por $H$. muscae, com a carga global média de $88,7 \%$ parasitos.

Pandey et al. ${ }^{1 h}$ (1981) assinalaram a presença de 11. muscae em $95,8 \%$ e $H$. majus (= H. microstoma) em $75,6 \%$ dos 94 eqüinos examinados no Marrocos, com o número médio de 576 espécimes de Habronema spp., dos quais cerca de $13 \%$ eram de $H$. majus e $87 \%$ de $H$. muscae. 
Lyons el al. ${ }^{2}$ (1983) registraram, em 363 eqüinos PuroSingue, no Kentucky, a ocorrência de $H$. muscac em $38 \%$. Ilabronema (formas imaturas) em 24\%, D. megastoma em $62 \%$ (formass adultas) e $13 \%$ com formas imaturas, com as intensidades médias de 46 (1-1.084) para H. muscae, de 122

\section{Tabela 1}

Ocorrências de Habronema muscae. H. microstoma e Draschia megastoma: pelos estados de origem dos eqüideos. Ceará e Minas Gerais, 1992/1993.

\begin{tabular}{|c|c|c|c|c|c|c|c|c|c|c|c|c|c|c|c|c|c|c|}
\hline \multirow{3}{*}{$\begin{array}{l}\text { A } \\
\text { n } \\
\text { i } \\
\text { n } \\
\text { i } \\
\text { i }\end{array}$} & & \multicolumn{17}{|c|}{ Estados de Origem } \\
\hline & \multirow[t]{2}{*}{$\begin{array}{l}\text { ra- } \\
\text { si- } \\
\text { tos }\end{array}$} & \multicolumn{2}{|c|}{$\begin{array}{l}\text { Yarn } \\
\text { nhdo }\end{array}$} & \multicolumn{2}{|c|}{ Plaui } & \multicolumn{2}{|c|}{ Coarí } & \multicolumn{2}{|c|}{$\begin{array}{c}\text { Rin } \\
\text { Cirande } \\
\text { Morte }\end{array}$} & \multicolumn{3}{|c|}{ Paralha } & \multicolumn{2}{|c|}{$\begin{array}{c}\text { Permambu } \\
\text { co }\end{array}$} & \multicolumn{2}{|c|}{$\begin{array}{l}\text { Minas } \\
\text { Cierals }\end{array}$} & \multicolumn{2}{|c|}{ Gờn } \\
\hline & & l: & $\mathrm{P}$ & $\mathrm{I}:$ & P & $\mathrm{T}$ & p & $\mathrm{T}$ & 1 & $\mathrm{I}$ & & $P$ & $\mathrm{~T}$ & $\bar{P}$ & $\mathrm{~T}$ & $\mathrm{P}$ & $1:$ & $P$ \\
\hline $\begin{array}{l}\mathbf{E} \\
\mathbf{Y}\end{array}$ & $\begin{array}{l}\text { II. } \\
\text { muscac }\end{array}$ & 3 & 2 & 1 & 3 & 1 & 4 & 1 & 2 & 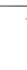 & & 2 & 2 & 2 & 13 & 3 & 3 & 1 \\
\hline ï & $\begin{array}{l}\text { II. mi - } \\
\text { crostoma }\end{array}$ & 3 & 2 & 4 & 3 & -1 & 2 & $t$ & 2 & . & & 2 & 2 & 1 & 13 & 1 & 3 & 0 \\
\hline n & $\begin{array}{c}\text { D. me- } \\
\text { gastoma }\end{array}$ & 3 & 0 & 4 & () & 4 & () & .4 & 1 & . & & 0 & 2 & () & 13 & 0 & 3 & 0 \\
\hline A & $\begin{array}{c}\text { H. } \\
\text { muscae }\end{array}$ & j & 0 & 4 & i & 3 & 3 & 3 & "is & 2 & & 0 & 3 & 3 & 2 & 1 & 10 & - \\
\hline $\begin{array}{l}\mathbf{i} \\
\mathbf{n}\end{array}$ & $\begin{array}{l}\text { H mi - } \\
\text { crustoma }\end{array}$ & 1 & I & 4 & ] & 3 & 3 & 3 & 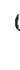 & 2 & & 0 & 3 & 2 & 2 & 1 & 0 & - \\
\hline $\begin{array}{l}\mathbf{i} \\
\mathbf{n} \\
\mathbf{0}\end{array}$ & $\begin{array}{l}\text { D. me- } \\
\text { gastoma }\end{array}$ & I & 0 & 4 & ] & 3 & 0 & 3 & i & 2 & & 0 & 3 & 0 & 2 & 0 & 0 & - \\
\hline $\begin{array}{l}\mathbf{M} \\
\mathbf{u}\end{array}$ & $\underset{\text { muscac }}{\mathbf{H} .}$ & 3 & I & 3 & 2 & 3 & 3 & 1 & 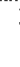 & 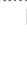 & & 1 & 4 & 3 & 5 & 1 & 2 & 1 \\
\hline $\mathbf{a}$ & $\begin{array}{l}\text { H. mi - } \\
\text { crostoma }\end{array}$ & 3 & I & 3 & I & 3 & 2 & I & 1 & & & I & 4 & 2 & 5 & () & 2 & (1) \\
\hline $\mathbf{r}$ & $\begin{array}{c}\text { D. me- } \\
\text { gastoma }\end{array}$ & 3 & 0 & 3 & ] & 3 & 0 & I & & & & 0 & 4 & 0 & 5 & 0 & 2 & (0) \\
\hline
\end{tabular}

$E=$ animais examinados; $P=$ positivos

\section{Tabela 2}

Habronema muscae, H. microstoma e Draschia megastoma: prevalências, intensidade das infecções, amplitude, total das infecções e relações macho/fêmea dos parasitos em eqüideos. Ceará e Minas Gerais, 1992/1993

\begin{tabular}{|c|c|c|c|c|}
\hline Hospedeiros & Parasitos & $\begin{array}{c}\text { Prevalência } \\
\%\end{array}$ & Intensidade Media & $\begin{array}{c}\text { Relaçāo } \\
\text { Macho /Fêmea }\end{array}$ \\
\hline & $\begin{array}{c}\text { H. } \\
\text { muscae }\end{array}$ & 51.35 & $\begin{array}{c}225,53 \\
(2-1206)\end{array}$ & $\begin{array}{c}1: 1,99 \\
(1: 1.04-130,5)\end{array}$ \\
\hline Eqüinos & H. microstoma & 35.13 & $\begin{array}{c}17.77 \\
(2-55)\end{array}$ & $\begin{array}{c}1: 1,46 \\
(1: 0,69-1: 38,0)\end{array}$ \\
\hline (37) & D. megustoma & - & - & - \\
\hline $\begin{array}{c}\text { Asininos } \\
\text { (18) }\end{array}$ & $\begin{array}{c}\text { H. } \\
\text { muscae } \\
\text { H. microstoma } \\
\text { D. megastoma }\end{array}$ & $\begin{array}{l}55.56 \\
44.44 \\
5.55\end{array}$ & $\begin{array}{c}44.6 \\
(1-171) \\
31,13 \\
(1-79) \\
5.0\end{array}$ & $\begin{array}{c}1: 1.95 \\
(1: 1,16-1: 4,17) \\
1: 1.52 \\
(1: 0.42-1: 3,0) \\
1: 1.95\end{array}$ \\
\hline $\begin{array}{c}\text { Muares } \\
\text { (22) }\end{array}$ & $\begin{array}{c}\text { H. } \\
\text { muscac } \\
\text { H. microstoma } \\
\text { D. megastoma }\end{array}$ & $\begin{array}{l}59.09 \\
11.95\end{array}$ & $\begin{array}{c}227.77(1-1203) \\
32.88(2-107) \\
1.0 \\
+\end{array}$ & $\begin{array}{c}1: 1.51 \\
(1: 0,94-1: 2,24) \\
1: 1.41 \\
(1: 0,5-1: 2,66) \\
1: 1,0\end{array}$ \\
\hline
\end{tabular}

(10-1.410) para Habronema spp. (formas imaluras) e de 92 (I0-930) para D. megastoma (formas imaturas).

Reinemeyer el al. ${ }^{17}$ (1984) assinalaram a presença de $/ 1$ muscae em $71 \%$ dos 55 cavalos necropsiados em Ohio State University (USA), com a intensidade média de 504 vermes e D). megastoma em $47 \%$, com a intensidade médial de 155.

Pandey; Eysker ${ }^{1 \downarrow}(1988)$ registraram a presençal de $H$. muscac em $85,7 \%$ dos 14 jumentos examinados no Zimbábue, com a intensidade média de 355 e $/ 1$. majus em 64,29\% com a intensidade médial de 272 e $D$. mégastoma em $78.5 \%$, com a intensidade média de 361 parasitos.

Kulkarni et al." (1989), em Bangalore, verificaram que $89,29 \%$ e 28 carcaças de eqüinos apresentavam nódulos de I). megastoma.

Eysker; Pandey" (1990)) assinalaram II. muscae em cinco cavalos no Zimbábue. com a média de 442 vermes por cavalo.

Pandey; Eysker ${ }^{15}(1990)$. lambém no Zimbábuc, encontraram $/ /$. muscae em $85,7 \%$, II. majus em $64,3 \%$ e 1$)$. megastoma em $78,6 \%$ dos jumentos examinados e formas jovens de espirurídeos em $78.6 \%$.

Lyons el al." (1992) apresentaram dados de vários levantamentos realizados no Kentucky, cobrindo o período 1951 a 1990 , onde $/ 1$. mascae aparece em preval lêncials que variaram de $3 \%$ a $73 \%$ e 1 . megastoma do zero at $63 \%$.

No Brasil, segundo Costa et al. (1986), há registros de D. megastoma em cavalos nos estados de Mato Grosso, Minas Gerais, Rio G. do Sul, Rio de Janeiro e São Paulo: $1 /$. microstoma $\mathrm{cm}$ calvalos de Malto Grosso. Distrilo Federal, Minas Gerais, Paraná, Pernambuco, Rio G. do Sul, Rio de Janeiro 
e São Paulo e em jumentos da Bahia; e $H$. muscae em cavalos do Distrito Federal, de Mato Grosso, Minas Gerais, Paraná, Pernambuco, Rio Grande do Sul, Rio de Janeiro e São Paulo.

Em vista disso, o objetivo deste estudo foi investigar as ocorrências e as intensidades médias das infecções ocasionadas por espécies dos gêneros Habronema e Draschia em asininos, muares e equiinos de alguns estados brasileiros.

\section{MATERIAL E MÉTODO}

Foram examinados os estômagos de três equiinos, um asinino e três muares do Maranhão, quatro equiinos, quatro asininos e três muares do Piauí, quatro eqüinos, três asininos e três muares do Ceará, quatro eqüinos, três asininos e um muar do Rio Grande do Norte, quatro eqüinos, dois asininos e um muar da Paraíba, dois eqüinos, três asininos e quatro muares de Pernambuco, treze equiinos, dois asininos e cinco muares de Minas Gerais e três equiinos e dois muares de Goiás, perfazendo os totais de 37 equiinos, 18 asininos e 22 muares. Cada estômago foi cuidadosamente aberto dentro de um balde e teve sua mucosa lavada, raspada e novamente lavada, sendo esta operação repetida duas vezes. O material recolhido no balde era deixado sedimentar, eliminando-se o sobrenadante e o sedimento era fixado a quente com solução de formalina a $10 \%$ e posteriormente acondicionado em saco plástico, com a devida identificação. Nestas condições foi transportado para o laboratório onde, com o auxílio de pinças, estiletes e pincéis, sob microscópio estereoscópico, procedeu-se à separação dos helmintos, transferindo-os para frascos tipo penicilina, necessariamente identificados. Para os estudos de taxonomia ao microscópio óptico, os helmintos foram diafanizados com lactofenol de Aman.

$O$ desvio padrão acompanhou a variação da média. Por esta razão, a análise de variância usada para a comparação das médias utilizou a transformação logarítmica $x=\log$ (número de helmintos contados mais um) por animal (Snedecor; Cochran ${ }^{14}$, 1968).

\section{RESULTADOS}

Os registros de ocorrência de $H$. muscae, $H$. microstoma e D. megastona encontram-se na Tab. I. Na Tab. 2 são apresentadas as prevalências, as intensidades médias, as amplitudes das infecções e as relações macho/fêmea para estes parasitos, nos três hospedeiros.

A análise de variância revelou não serem significativamente diferentes as médias das infecções em equiinos e asininos, tanto para $H$. muscae quanto para $H$. microstoma $(\mathrm{p}=$ 0,7012 e $p=0,3141$, respectivamente); na comparação eqüino/muar $(\mathrm{p}=0,4234$ e $\mathrm{p}=0,6874$, respectivamente para $\boldsymbol{H}$. muscae e para $H$. microstona) e, ainda, na comparação asinino/muar $(p=0,6886$ e $p=0,6377$, respectivamente para
H. muscae e para $H$. microstoma).

\section{DISCUSSĀO}

Os espirurídeos de eqüídeos têm sido constante motivo de preocupação pela gravidade das lesões que ocasionam: gastrites, formações tumorais e habronemose cutânea (Kulkarni et al." 1989; Blagburn et al. ${ }^{2}, 1991$; Lyons et al. ${ }^{10}$, 1991), podendo resultar em morte do animal (Costa; Freitas $^{4}, 1963$ ).

Os dados de prevalência da Tab. 2 sugerem ser $H$. musc'ac a espécie mais comum nos três hospedeiros: $51,35 \%$ em equuinos, $55,56 \%$ em asininos e 59,09\% em muares, o que é concordante com as observações de Foster; Ortiz ${ }^{7}$ (1937) em eqüinos no Panamá, de Waddell ${ }^{20}$ ( 1969 ) em eqüinos na Austrália, de Graber ${ }^{8}$ (1970) em asininos e equiinos no Tchad, de Pandey et al..$^{16}$ (1981) em eqüinos no Marrocos, de Reinemeyer et al. ${ }^{17}$ (1984) em eqüinos de Ohio, USA e de Pandey; Eysker ${ }^{14}$ (1988) em asininos no Zimbábue. A espécie $D$. megastoma não foi registrada nos equiinos necropsiados e os registros em asininos e muares foram de baixas prevalências, respectivamente $5,55 \%$ e $9,09 \%$, todos eles com baixa intensidade parasitária.

A intensidade média das infecções também foi maior para $H$. muscale, nos três hospedeiros, respectivamente 226 , 45, 228 para eqüinos, asininos e muares, contra 18,31 e 33 para $H$. microstoma, nos mesmos hospedeiros, fato também registrado por Foster; Ortiz (1937) em cavalos e Graber $^{*}(1970)$ em cavalos, muito embora o inverso possa ocorrer, como registraram Pandey; Eysker ${ }^{14}$ (1988) em jumentos. Quanto a D. megastoma, embora tenha sido registrada em baixas prevalências e em baixas intensidades de infecções, é espécie muito importante em outras regiões, como o demonstraram Graber ${ }^{8}$ (1970); Alcaino ef al.' (1980); Lyons et al. ${ }^{2}$ (1983): Reinemeyer el al. ${ }^{17}$ (1984); Pandey; Eysker ${ }^{14}$ ( 1988); Kulkarni et al. ${ }^{\prime}$ (1989) e Lyons et al." (1992).

Apesar de aparentes diferenças em alguns casos, a análise estatística demonstrou não serem significantemente diferentes as intensidades médias de $H$, muscae e de $H$. microstoma nos três hospedeiros.

Como tem sido habitual em outras espécies de nematóides parasitos de animais domésticos, as relaçōes macho/fêmea evidenciam que as fêmeas de $H$. nuiscae e de H. microstona ocorrem em maior número que os machos, nos três hospedeiros.

\section{AGRADECIMENTOS}

Ao Prof. Sylvio Barbosa Cardoso e ao centro de Ciências da Saúde, da Universidade do Ceará pelas facilidades oferecidas para a realização de necrópsias. Agradecemos, também, a Mariângela Valente, a Sumara Guimarães Cavalcante e a Marco Túlio C. Oliveira, na ocasião estudantes de Medicina Veterinária, pela ajuda durante as necrópsias. 


\section{SUMMARY}

Thirty-seven Equus caballus, 18 Equus asinus and 22 mules from the States of Maranhão, Piauí, Ceará, Rio Grande do Norte, Paraíba, Pernambuco, Minas Gerais and Goiás had their stomachs examined to collect spirurid worms, and the following species were found: Draschia megastoma (Rudolphi, 1819), Habronema muscae (Carter, 1861) and Habronema microstoma (Schneider, 1866). Prevalences, mean intensities and Male/Female ratios are considered. The variance analyses showed that no significant statistical differences ocurred among the mean intensities of $H$. muscae and $H$. Microstoma.

UNITERMS: Habronema; Draschia; Equidae; Mules; Brazil.

\section{REFERENCES}

1-ALCAINO, H.; GORMAN, T.; CONEJO, S.; MOLINARI, R.; PINTO, A. Estudo epizootiológico de parasitismo estomacal del eqüino de la zona Centro Sur de Chile, Archives Medicine Veterinary, v.12, p.10-29, 1980.

2-BLAGBURN, B.L.; HENDRIX, C.M.; LINDSAY, D.; SCHUMACHER, J Pathogenesis. treatment and control of gastric parasites in horses. Compendium on Continuing Education. v. 13, p.850-8. 1991.

3-CHRISTENSEN, N.O.: ROTH. H. On parasitic gastritis in the horse. due to Trichostrongylus axei (Cobb.. 1879) Railliet \& Henry, 1909. Acta Pathologica et Microbiologica Scandinavica, v.19, p.310-1, 1942

4-COSTA. H.M.A.: FREITAS, M.G. Comunicação sobre novos achados helmintológicos em animais domésticos de Minas Gerais. Arquivos da Escola de Veterinária da Universidade Federal de Minas Gerais, Belo Horizonte, v. 15, p.35-40, 1963

5-COSTA. H.M.A.; LEITE, A.C.R.; GUIMARÃES, M.P.; LIMA. W.S. Distribuição de helmintos parasitos de animais domésticos no Brasil. Arquivos Brasileiros de Medicina Veterinária e Zootecnia. v.38. p.465-579, 1986.

6-EYSKER, M.: PANDEY, V.S. Observations on some parasites of the horse in Zimbabwe. Zimbabwe Veterinary Journal. v.21, p.21-4, 1990.

7-FOSTER, A.O.; ORTIZ, O.P. A Further report on the parasites of selected group of equines in Panama. Journal of Parasitology. v.23, p.360-4, 1937.

8-GRABER, M. Helminthes et helminthiases des equidés (ânes et chevaux) de la République du Tchad. Revue d'Elevage et de Médecine Vétérinaire des Pays Tropicaux, v.23, p.207-22, 1970.

(0)-KULKARNI, S.; VIJAYASARTHY, S.K.; JAGANNATH, M.S.; SRINIVASA GOWDA. R.N.: SESHADRI, S.J. A study on Draschia megastoma (Chitwood and Wehr, 1934) Infections in Horses. Indian Veterinary Journal, v.66, p.899-900, 1989.

10-L.YONS, E.T.; DRUDGE, J.H.; TOLLIVER, S.C. A large Draschia megastoma - related gastric lesions in a Thoroughbred. Veterinary Medicine, v.86, p.332-4, 1991.

II-LYONS, E.T.; DRUDGE, J.H.; TOLLIVER, S.C. Review of prevalence surveys of internal parasites recovered (1951-1990) from horses at necropsy in Kentucky. Journal Equine Veterinary Science. v.12, p.9-16, 1992

12-LYONS. E.T.: TOLLIVER, S.C.; DRUDGE. J.H.: SWECZEK, T.W.: CROWE, M.W. Parasites in Kentucky Thoroughbreds at necropsy: emphasis on stomach worms and tapeworms.

American Journal of Veterinary Research. v.44, p.839-44, 1983.

13-OUHELL1, H.; CABARET, V.S.; ELKHAFANE, A. Localisation of parasites in the stomach of horses of the region of Settat (Marocco) Revue d'Elevage et de Médecine Vétérinaire des Pays Tropicaux, v.32, p. 347-52, 1979

14-PANDEY, V.S.; EYSKER, M. Parasites of the stomach in donkeys of the Highveld of Zimbabwe. Veterinary Quartely, v.10, p.246-8. 1988.

15-PANDEY, V.S.; EYSKER, M. Internal parasites of donkeys from the Highveld of Zimbabwe. Zimbabwe Veterinary Journal, v.21, p.27-31, 1990
16-PANDEY, V.S.; OUHELLI, H.; ELKHAFANE, A. Epidemiological observations on stomach worms of horses in Marocco. Journal of Helminthology, v.55, p.155-60, 1981.

17-REINEMEYER, C.R.; SMITH, S.A.; GABEL, A.A.; HERD. R.P. The prevalence and intensity of internal parasites of horses in the USA. Veterinary Parasitology, v.15, p.75-83, 1984.

18-SCIALDO. R.C. A Survey of stomach parasites of horses from the Southwestern. Southern and Central States. Southwestern Veterinary. v.30, p.155-7, 1977

19-SNEDECOR, G.W.; COCHRAN, W.G. Statistical methods. Ames, Iowa State University Press, 1968.

20-WADDELL, A.H. A Survey of Habronema spp, and the identification of third-stage larvae of Habronema megastoma and Habronema muscae in section. Australian Veterinary Journal, v.45, p.20-1, 1969. 